\title{
Eddy Current Loss Calculation in Permanent Magnet of SPM Motor Including Carrier Harmonics Based on Reluctance Network Analysis
}

\author{
Y. Yoshida, K. Nakamura, and O. Ichinokura \\ Graduate School of Engineering, Tohoku University, 6-6-05 Aoba, Aramaki, Aoba-ku, Sendai 980-8579, Japan
}

\begin{abstract}
This paper presents a method for calculating eddy current loss in a permanent magnet (PM) of a surface permanent magnet (SPM) motor based on electric and magnetic networks. First, a reluctance network analysis (RNA) model of the SPM motor and coupling between the RNA model and electric network model are described. Then, the eddy current loss in the magnet of the SPM motor from no load to full load current conditions including and excluding carrier harmonics are calculated by the proposed model. Finally, it is demonstrated that the RNA model can be taken the effect of division of the magnet into consideration to reduce the eddy current loss in the magnet.
\end{abstract}

Key words: Reluctance network analysis (RNA), surface permanent magnet (SPM) motor, eddy current loss

\section{Introduction}

In recent years, design and analysis of permanent magnet (PM) motors considering magnetic nonlinearity and eddy current loss in rare-earth magnet becomes important since the working flux density of the PM motors tends to be high due to high power-to-weight ratio, and the $\mathrm{PM}$ motors are driven by a PWM inverter with high frequency switching.

The most conventional optimum design for PM motors is shape optimization by finite element analysis (FEA). However, to calculate the eddy current loss in the magnet, three-dimensional (3-D) FEA should be used and it takes much time to obtain the calculation results in general. Therefore, more practical solution for the PM motor analysis in reasonable time with high accuracy is strongly required.

Reluctance network analysis (RNA) can be useful for saving calculation time to estimate characteristics of PM motors. It can be taken into account the magnetic saturation and the rotor motion ${ }^{1)-6)}$.

In previous papers, we have proposed a method for calculating characteristics of PM motors based on RNA $^{1)-3)}$. The proposed RNA model of the PM motor can be coupled with the motor drive circuit and mechanical system. Using the proposed model, iron loss in the stator core of the PM motor was calculated accurately2). In addition, eddy current loss in permanent magnets of a surface permanent magnet (SPM) motor can be calculated by coupling with an electric network model of the magnet ${ }^{7)}$. However, the eddy current loss in the magnets caused by carrier harmonics was not discussed.

This paper describes a method for calculating the eddy current loss of the magnet including carrier harmonics based on RNA. In order to prove the validity of the proposed model, calculated values are compared with ones obtained from the 3-D FEA.

\section{Analysis model of SPM motor}

Fig. 1 shows shape and specifications of an SPM motor used in the consideration. The material of the magnet is sintered $\mathrm{Nd}-\mathrm{Fe}-\mathrm{B}$. The coercive force $H_{c}$ and the recoil permeability $\mu_{r}$ are $920 \mathrm{kA} / \mathrm{m}$ and 1.038 , respectively.

The eddy current loss in the magnets is increasing when the PM motor is driven by PWM inverter because the magnets are exposed by carrier harmonics due to high frequency switching. Therefore, in this study, the eddy current loss in the magnets caused by the carrier harmonics is evaluated based on RNA.

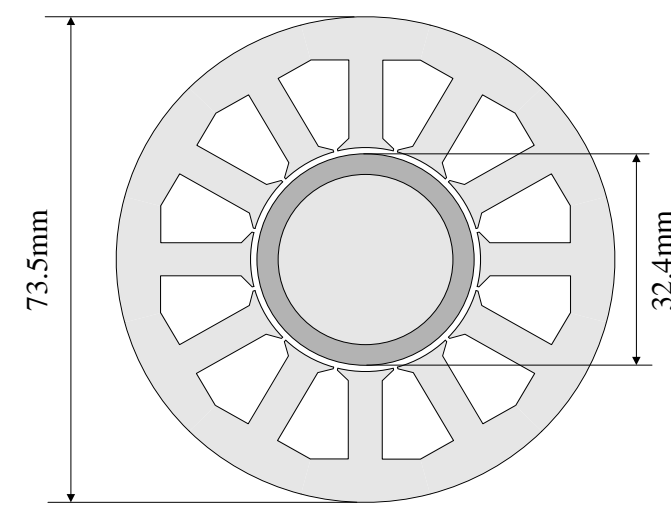

\begin{tabular}{c|c}
\hline Number of slots & 12 \\
\hline Number of poles & 10 \\
\hline Number of winding turns / slot & 37 \\
\hline Air-gap & $0.7 \mathrm{~mm}$ \\
\hline Stack length & $64 \mathrm{~mm}$ \\
\hline Core material & $35 \mathrm{~A} 300$ \\
\hline Permanent magnet material & $\mathrm{Nd}-\mathrm{Fe}-\mathrm{B}$ \\
\hline Resistivity of magnet & $1.16 \times 10^{-6} \Omega \cdot \mathrm{m}$ \\
\hline
\end{tabular}

Fig. 1 Shape and specifications of SPM motor. 


\section{Proposed calculation method}

Eddy current loss in the magnets of the SPM motor can be calculated by the RNA model and electric network model ${ }^{7}$. In this section, the RNA of the SPM motor and the electric network model for calculating the eddy current loss are mentioned.

\subsection{RNA model of SPM motor}

The SPM motor is divided into multiple elements taking into consideration the motor shapes and flux flow. Since the flux flowing through the SPM motor is uniform distribution in the axial direction, the RNA model is not divided in the axial length. Thus, each divided element is expressed in a two-dimensional (2-D) unit magnetic circuit. Fig. 2 shows a part of RNA model. Each element in air gap is divided in one degree intervals in circumferential direction and the magnets are divided into three in radial direction as shown in the figure.

\subsection{Electric network model}

Fig. 3(a) shows an example of a conductor which is divided into multiple elements. Each divided element is expressed in electric resistances and electromotive forces (EMFs). By connecting the electric resistances and EMFs of all branches together, an electric network model for calculating the eddy current can be obtained as shown in Fig. 3(b).

The resistances in the $x$-axis direction $R_{X}$ and in the $z$ axis direction $R_{Z}$ in each element are obtained from

$$
\begin{aligned}
& R_{X}=\rho \frac{2 \Delta X}{\Delta Z Y}, \\
& R_{Z}=\rho \frac{2 \Delta Z}{\Delta X Y},
\end{aligned}
$$

where $\rho$ is the resistivity, $\Delta X$ is the element length in the $x$-axis direction, $\Delta Z$ is the element length in the $Z$ axis direction and $Y$ is the conductor thickness.

The EMF is induced when the flux passing through the element changes with time based on Faraday's law. The EMF of the $k^{\text {th }}$ element is given by

$$
e_{k}=-\frac{\partial B_{y k}}{\partial t} \Delta X \Delta Z
$$

where the flux density of the $k^{\text {th }}$ element is $B_{y k}$.

If equipotential lines in the conductor can be expressed in radial lines as shown in Fig. 4, the potential difference $V_{A B}$ between node $\mathrm{A}$ and node $\mathrm{B}$ in the figure is expressed by

$$
V_{A B}=e_{k} \frac{\theta_{B}-\theta_{A}}{2 \pi},
$$

where $\theta_{A}$ is the angle between the reference line and the equipotential line passing through node $\mathrm{A}$, and $\theta_{B}$ is the angle between the reference line and the equipotential line passing through node B.
Thus, the induced voltage in the $j^{\text {th }}$ branch, which is produced by $B_{y k}$ flowing through the $k^{\text {th }}$ element, is obtained from

$$
V_{k j}=e_{k} \frac{\theta_{k j}}{2 \pi},
$$

where $\theta_{k j}$ is the angle between the equipotential lines passing through the both ends of the $j^{\text {th }}$ branch (in the case of Fig. $\left.4, \theta_{k j}=\theta_{B}-\theta_{A}\right)$.

The induced voltage of each branch can be calculated from the summation of the induced voltages caused by the flux flowing through all elements. Therefore, the induced voltage on each branch $V_{j}$ is

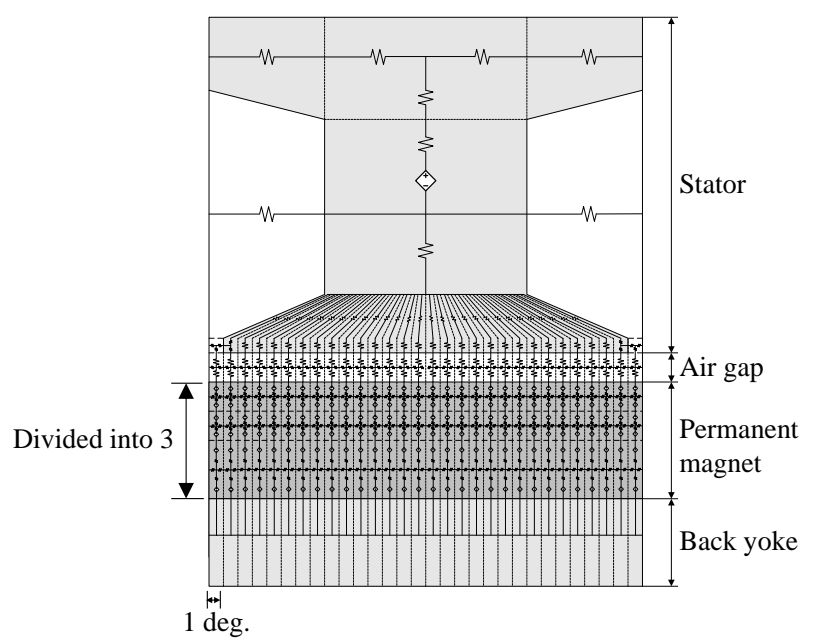

Fig. 2 A part of RNA model of the SPM motor.

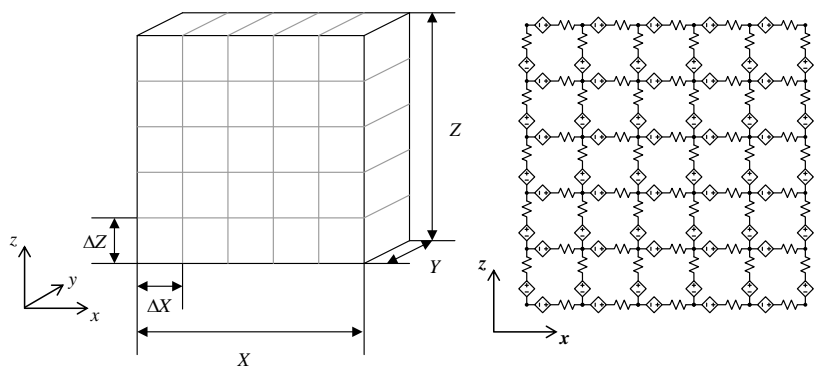

(a) Division of a conductor (b)Electric network model Fig. 3 An example of division of a conductor and electric network model for calculating the eddy current loss.

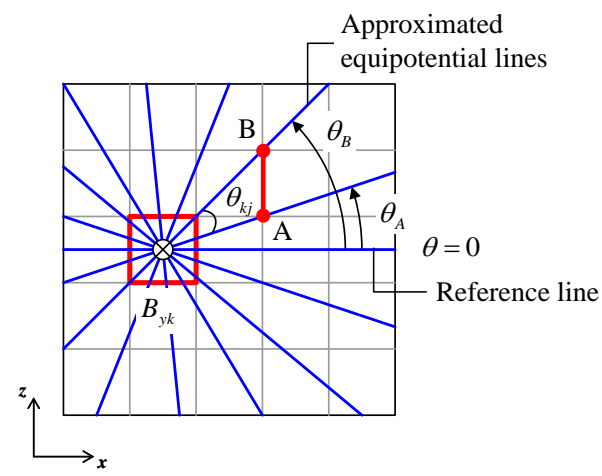

Fig. 4 Equipotential lines of induced voltage generated in one element. 


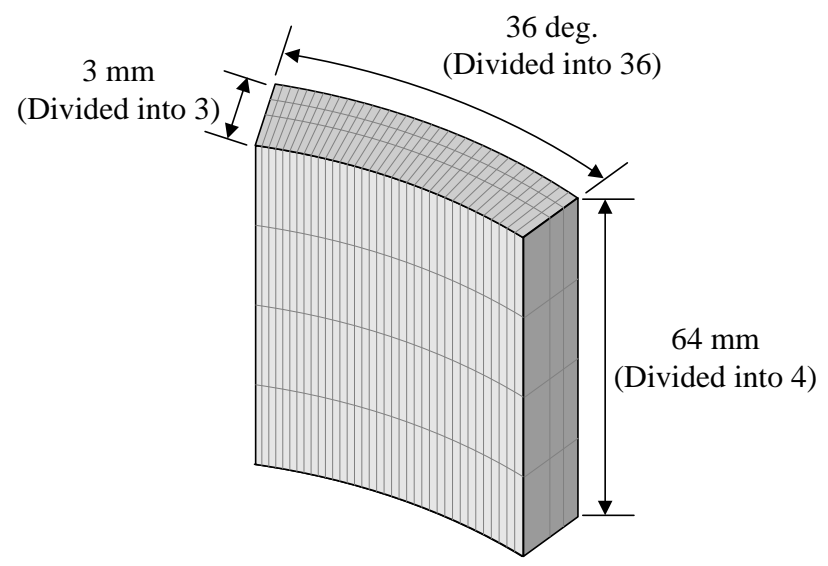

Fig. 5 Shape of one pole of the magnet and its division for the electric network model.

given by

$$
V_{j}=\sum_{k=1}^{K} e_{k} \frac{\theta_{k j}}{2 \pi}
$$

where $K$ is the number of elements.

Fig. 5 shows the shape and division of one pole of the magnet to derive the electric network model for calculating the eddy current loss. In the figure, the magnet is divided into three in the radial direction, 36 in the circumferential direction, respectively. In the axial direction, the magnet is divided into four.

\section{Eddy current loss estimation in PM of SPM motor}

The eddy current loss in the magnet taking account of the carrier harmonics are calculated by the proposed model and compared with 3-D FEA results.

\subsection{Carrier frequency characteristics}

The eddy current loss in the magnet corresponding to various carrier frequencies is calculated to investigate the influence of the carrier harmonics.

To take into account the carrier harmonics, triangular waves are added to the sinusoidal waves. Fig. 6 shows phase current including carrier harmonics. Frequency and amplitude of the triangular wave are $4.5 \mathrm{kHz}$ and $0.5 \mathrm{~A}$, respectively while the fundamental frequency is $250 \mathrm{~Hz}$.

Fig. 7 provides the carrier frequency versus eddy current loss in one pole of the magnet at an input current amplitude of $5 \mathrm{~A}$. In this figure, the calculated value of $0 \mathrm{kHz}$ is obtained when the input current is sinusoidal. It is understood that the eddy current loss in the magnets increases in proportion to the carrier frequency. Moreover, the difference between the proposed model and 3-D FEA becomes larger as the carrier frequency increases, since the proposed model does not take the magnetic reaction field caused by the eddy current into account yet.

Fig. 8 shows the relationship between the eddy current loss in one pole of the magnet and phase current. As shown in this figure, the calculated values obtained from the proposed model exhibits good matches with the ones obtained from 3-D FEA when the SPM motor is driven by the ideal sinusoidal current. The eddy current loss becomes about three times larger at a rated phase current of $5 \mathrm{~A}$ when the carrier harmonics is added to the phase current. Thus, it is clarified the carrier harmonics cause eddy current loss increase in the magnet of the SPM motor.

\subsection{Effect of magnet division}

For a countermeasure against the eddy current loss, it is effective to divide the magnet into several pieces. Fig. 9 shows the relationship between the number of magnet divisions and the eddy current loss in the magnets at a phase current of $5 \mathrm{~A}$ with $4.5 \mathrm{kHz}$ carrier frequency. The magnets are divided in the axial direction. It is clear that the eddy current loss in the magnet can be reduced by dividing the magnet. Fig. 10 shows the eddy current density distribution obtained from the proposed model. As shown in this figure, the eddy current density at the edge of the magnets can be reduced as the number of divisions increase.

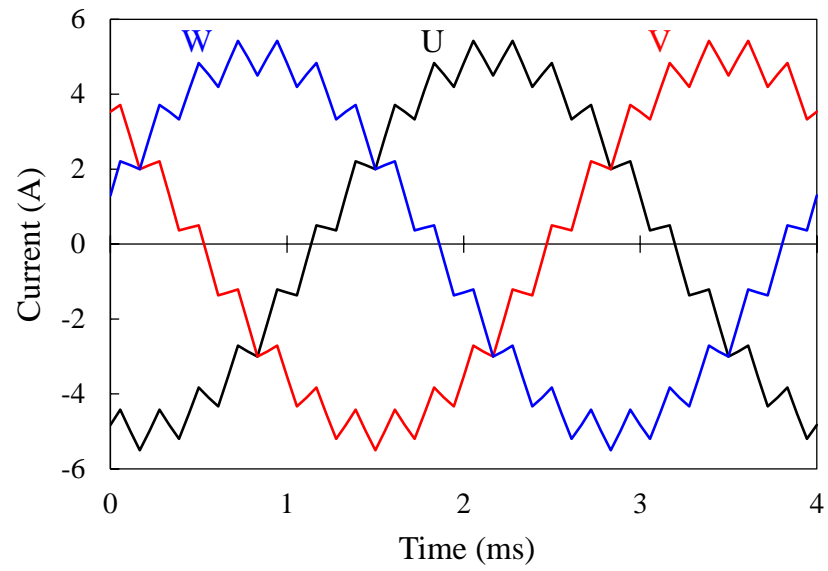

Fig. 6 Input phase current waveforms with $4.5 \mathrm{kHz}$ triangular waves as including carrier harmonics at a amplitude of $5 \mathrm{~A}$.

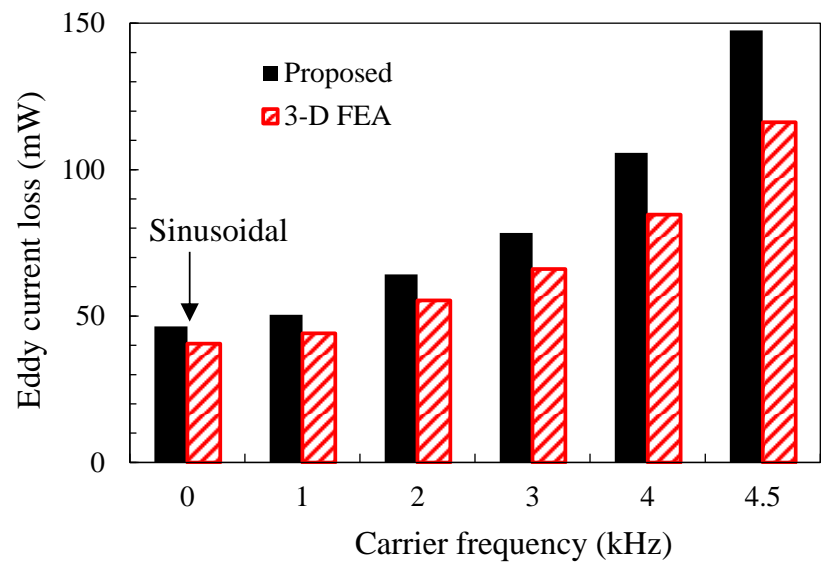

Fig. 7 Carrier frequency versus eddy current loss characteristics. 


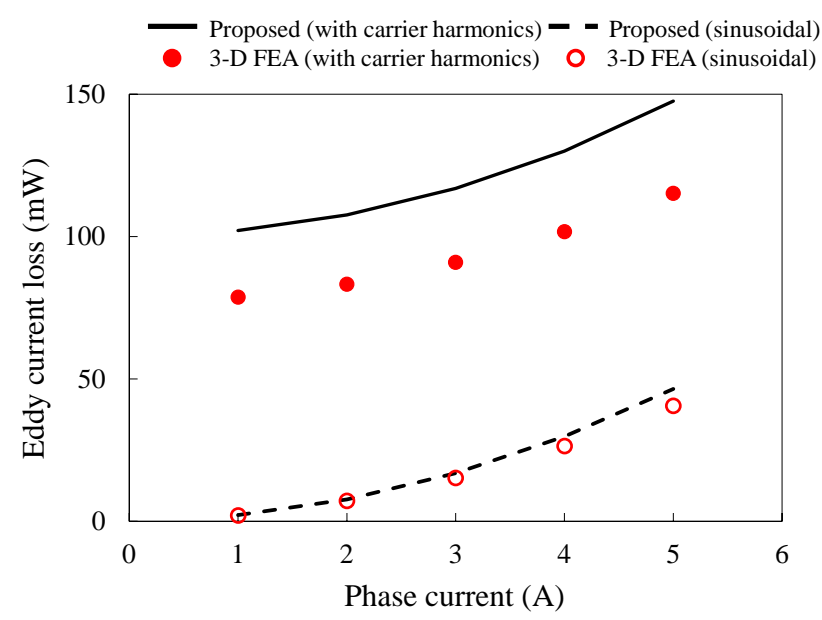

Fig. 8 Comparison of eddy current loss in the magnet between including and excluding carrier harmonics.

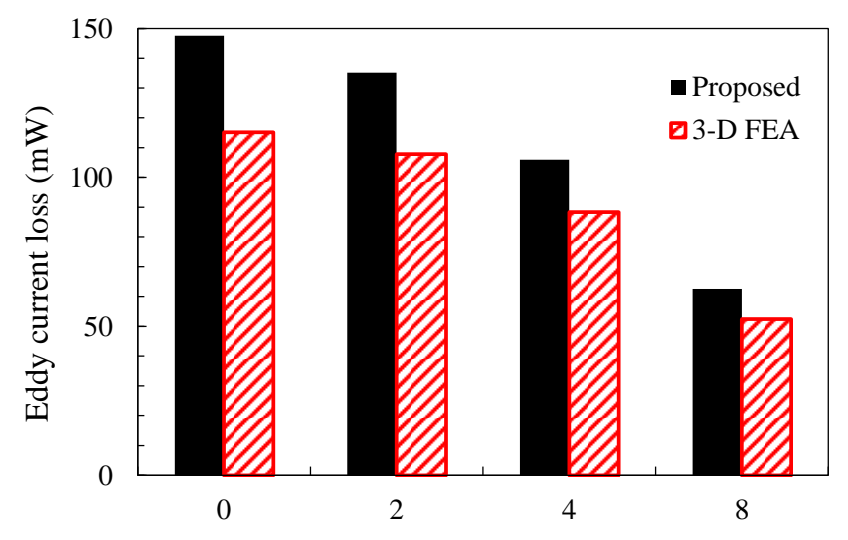

The number of divisions in the axial direction

Fig. 9 Relationship between the number of divisions and eddy current loss in the magnet.
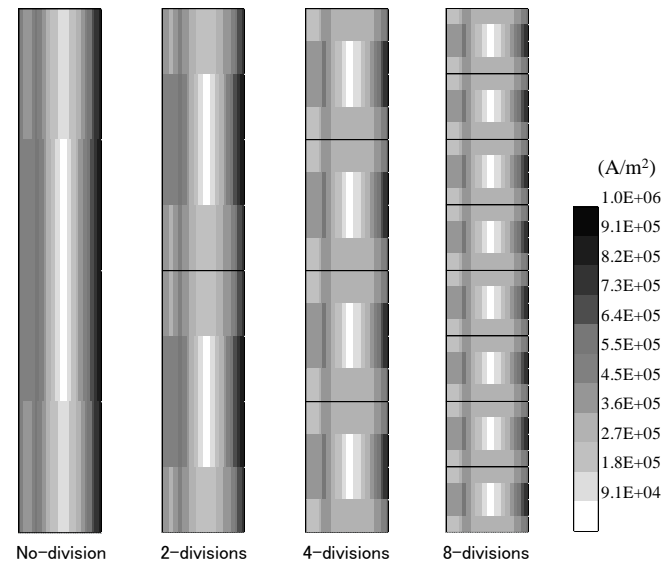

Fig. 10 Eddy current density distribution in the magnet obtained from the proposed model.
Table 1 Comparison of calculation time.

\begin{tabular}{|c|c|c|}
\hline & Number of elements & Calculation time \\
\hline RNA (full model) & 1824 & 20 minutes \\
\hline 3D-FEA (1/4 model) & 75249 & 8 hours \\
\hline
\end{tabular}

\subsection{Calculation time}

Table 1 gives the comparison of the calculation time and the number of elements between the RNA model and 3-D FEA model. The calculation time to obtain the eddy current loss at full load condition by RNA is 20 minutes, while the 3 -D FEA requires 8 hours for the same condition.

\section{Conclusion}

This paper presents a method for calculating eddy current loss in the permanent magnet of the SPM motor based on electric and magnetic networks. The proposed model can express the influence of the carrier harmonics and the effectiveness of division of the magnet to reduce the eddy current loss. It is conceivable that the difference between the proposed method and 3-D FEA becomes larger when the carrier harmonics is added to the phase current because the effect of the magnetic reaction field caused by the eddy current increases. Therefore, further studies will attempt to develop the RNA model considering the magnetic reaction field by using magnetic inductance in the RNA model ${ }^{8}$.

\section{References}

1) K. Nakamura, K. Saito, O. Ichinokura: IEEE Trans. Magn., 39, 3250 (2003).

2) K. Nakamura, M. Ishihara, O. Ichinokura: 17th International Conference on Electrical Machines (ICEM 2006), PSA1-16 (2006).

3) K. Nakamura, O. Ichinokura: 13th International Power Electronics and Motion Control Conference (EPE-PEMC 2008), 441 (2008).

4) T. Raminosoa, J.A. Farooq, A. Djerdir, A. Miraoui: Energy Conversion and Management, 50, 1356 (2009).

5) J.K. Tangudu, T.M. Jahns, A.M. EL-Refaie, Z.Q. Zhu: Energy Conversion Congress and Exposition (ECCE 2009), pp. 2423 2430 (2009)

6) Seok-Hee Han Thomas M. Jahns Wen L. Soong: International Electric Machines and Drives Conference (IEMDC 2007), 1, 21 (2007).

7) Y. Yoshida, K. Nakamura, O. Ichinokura: IEEE Trans. Magn., 48, 3108 (2012).

8) Y. Yoshida, K. Nakamura, O. Ichinokura: IEEE Trans. Magn., 47, 4155 (2011).

Received September 19, 2012; Revised November 24, 2012; Accepted January 7, 2013 